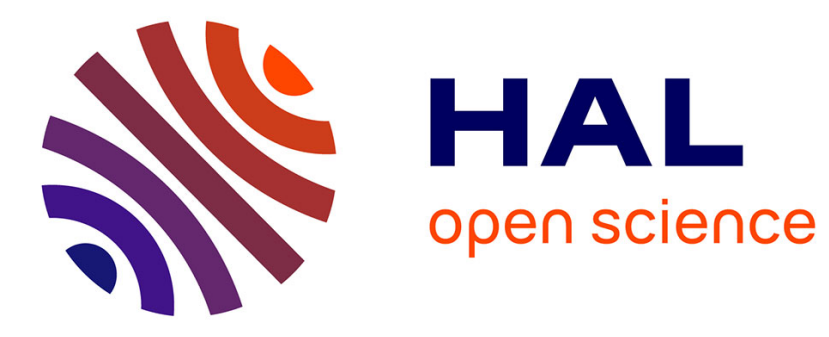

\title{
Understanding User Strategies When Touching Arbitrary Shaped Objects
}

\author{
Quentin Roy, Simon Tangi Perrault, Katherine Fennedy, Thomas Pietrzak, \\ Anne Roudaut
}

\section{- To cite this version:}

Quentin Roy, Simon Tangi Perrault, Katherine Fennedy, Thomas Pietrzak, Anne Roudaut. Understanding User Strategies When Touching Arbitrary Shaped Objects. Proceedings of the ACM International Conference on Mobile Human-Computer Interaction (MobileHCI 2021), Sep 2021, Toulouse, France. 10.1145/3447526.3472038 . hal-03272566

\section{HAL Id: hal-03272566 https://hal.inria.fr/hal-03272566}

Submitted on 28 Jun 2021

HAL is a multi-disciplinary open access archive for the deposit and dissemination of scientific research documents, whether they are published or not. The documents may come from teaching and research institutions in France or abroad, or from public or private research centers.
L'archive ouverte pluridisciplinaire $\mathbf{H A L}$, est destinée au dépôt et à la diffusion de documents scientifiques de niveau recherche, publiés ou non, émanant des établissements d'enseignement et de recherche français ou étrangers, des laboratoires publics ou privés. 


\section{Understanding User Strategies When Touching Arbitrary Shaped Objects}

\author{
Quentin Roy \\ University of Waterloo \\ Waterloo, Canada \\ quentin@quentinroy.fr
}

\author{
Simon Tangi Perrault \\ Singapore University of Technology \\ and Design \\ Singapore, Singapore \\ simon_perrault@sutd.edu.sg
}

\author{
Katherine Fennedy \\ Singapore University of Technology \\ and Design \\ Singapore, Singapore \\ katherine_fennedy@mymail.sutd.edu.sg
}

\author{
Thomas Pietrzak \\ University of Lille \\ Lille, France \\ thomas.pietrzak@univ-lille.fr
}

\author{
Anne Roudaut \\ University of Bristol \\ Bristol, UK \\ anne.roudaut@bristol.ac.uk
}

\begin{abstract}
We investigate how users touch arbitrary shapes. First, we performed semi-structured interviews with a fifteen-shape set as prop to identify touch strategies. Results reveal four main potential touch strategies, from which we devised nine mathematical candidate models. We investigate the ability of these models to predict human behaviour in a controlled experiment. We found that the center of a shape's bounding box best approximates a user's target location when touching arbitrary shapes. Our findings not only invite designers to use a larger variety of shapes, but can also be used to design touch interaction adapted to user behaviour using our model. As an example, they are likely to be valuable for the creation of applications exposing shapes of various complexities, like drawing applications.
\end{abstract}

\section{CCS CONCEPTS}

- Human-centered computing $\rightarrow$ Human computer interaction (HCI); Empirical studies in HCI; Touch screens.

\section{KEYWORDS}

Touch, Accuracy, Non-Rectangular Widgets, Arbitrary Shapes

\section{ACM Reference Format:}

Quentin Roy, Simon Tangi Perrault, Katherine Fennedy, Thomas Pietrzak, and Anne Roudaut. 2021. Understanding User Strategies When Touching Arbitrary Shaped Objects. In . ACM, New York, NY, USA, 11 pages.

\section{INTRODUCTION}

Touch systems are populated with touchable objects, such as buttons, drawings, icons, links, or other widgets of many different shapes. However, despite this abundance of "touchable" object geometries within our interfaces, there is little known about where the users chose to touch them.

Being able to predict how users aim at arbitrarily shaped objects can help designers choosing better shapes for interactive elements, guide developers in implementing systems that better predict user's mental models and avoid errors, or bring to researchers insights on human touch behavior and expand our understanding of basic interactive mechanics. The quest for this knowledge is particularly true in the case of touch interaction because of the fat finger issue: as the finger is occluding a part of the interface during the interaction, it prevents users to aim precisely, thus encouraging them to guess the intended touch location.

Previous work tends to indicate the geometry of an object impacts how users attempt to touch it. For example, Grossman et al. [13] observed that users adopt different strategies in function of the shape of their target when pointing with a computer mouse. Roudaut et al. [19] demonstrated that touch accuracy is affected by the curvature of the touch surface. Finally, Holz and Baudisch [16] suggest that users rely on geometrical features of their fingertips when attempting to precisely touch a location indicated by a cross.

This paper extends this body of work, investigates user strategies when touching arbitrary shapes on touch screens, and proposes a model of users' behavior when touching arbitrary shapes. First, we performed semi-structured interviews with paper mock-ups during which we asked participants to touch various shapes and to describe their strategy in doing so. The analysis of their feedback revealed four different strategies for touch input. In particular, we identified behavior differences when the finger fully occluded the objects, or when it was only partially occluded. Second, we conducted a quantitative controlled experiment to empirically verify which model is the most representative of touch behavior on arbitrary shapes. More precisely, we designed a study with three particular characteristics:

- We devised nine candidate mathematical models that represent the four touch strategies highlighted in our first qualitative study. We moved from 4 to 9 because each qualitative strategy could be interpreted in multiple quantitative ways.

- We used a computational approach to automatically extract 15 arbitrary shapes out of a set of icons. We did this instead of choosing ad hoc shapes to maximize the difference between the prediction of our models and increase our chance to find a signal in the noise.

- We pick two sizes of shapes relative to the finger size, more precisely $25 \%$ larger than the participants' fingertips or $25 \%$ smaller. This is quite atypical for touch studies which 
commonly use absolute sizes. We did this to verify our observations that different strategies were used depending on whether the finger fully occludes the shape or not.

Our results showed that the center of a shape's bounding box best models how users touch it. This model is accurate even for asymmetrical shapes, or shapes including holes. Our work shows that designers can safely use a larger variety of geometries, without changing the touch detection algorithm since the bounding box model is used in many commercial systems. It also indicates that feedback indicating the interactive area may not be needed provided that it includes a disc of $4 \mathrm{~mm}$ radius centered on the center of the shape's bounding box. Our analysis also reveals that larger shapes induced more touch variations (spread) but less offset. This contradicts previous assumptions and observations that users align their fingertips with the geometrical features extending from under their fingers to point more systematically in the same locations over several trials.

To summarize, we contribute to the first set of studies investigating touch accuracy on arbitrarily shaped objects and looking at how users place their fingers onto them. Our contribution generalizes to any touchable elements. One example is games that provide several elements to be manipulated which are not icons; a shape inserted in a drawing; or a partially occluded letter in a text document. Understanding how end-users touch those elements is of interest to the community in that we can better model human behavior. Studies looking at touch accuracy had a major impact on HCI and consumer products. Our work extends them with arbitrary shapes and invites designers to use more diverse geometries of shapes within UIs.

\section{RELATED WORK}

We first define the type of study we used in this paper, i.e. touch accuracy. We then discuss the current understanding of the topic, including investigations of shapes with different geometries. We finally discuss the difference between touch accuracy and pointing studies.

\subsection{Touch Accuracy Studies: Definition and Metrics}

Touch accuracy studies have seen an increase in interest since the rise of touchscreen devices. Touch accuracy is indeed an intrinsic problem on touchscreen devices because of the fat finger problem $[18,22]$. In particular, the fingertip occludes the shapes and makes selection difficult and error-prone on touchscreens. Such studies are particularly focusing on the absolute precision of the finger and are using tasks in which the participants must point at a given object the most accurately possible without time constraints. These studies particularly focus on where the users are placing their fingers. They thus generally make use of an extra mechanism to let participants confirm their final touch location via another action such as using a foot switch $[15,16,19]$.

Within those works, touch accuracy is defined as the combination of three values: the offset, the spread, and the minimum button size [15]. Multiple targeting produces contact points, generally the center of gravity of points in the contact area. All contact points can thus be summarized as:
- Offset, the distance between the centroid of a cluster of points and the target ${ }^{1}$ which is typically a single point such as the center of the cross.

- Spread, the mean distance of all points in a population trial from their centroid.

- Minimum button size, the diameter of the smallest circular area that contains $95 \%$ of all point acquisitions.

Though the connection has not been made in the literature, the minimum button size defined in touch accuracy studies relates to effective width [17] used in pointing studies (see subsection "Pointing vs. touch accuracy studies"). The effective width is computed from the standard deviation in the selection coordinates gathered over a sequence of trials. Holz et al. $[15,16]$ used the $95 \%$ bracket of the standard distribution instead for defining the minimum button size. This is certainly because this value is closer to the actual meaning of the term: the minimum button size guarantees that the touches will be accurate $95 \%$ of the time. It means that accurate touch is still possible with smaller buttons, but the frequency of errors is higher.

\subsection{Touch Accuracy Studies: Current Knowledge}

2.2.1 Offset. Evidence has shown the existence of a systematic effect that causes a touch device to sense at an offset from a target. Benko et al. showed that the center of the contact area moves under pressure, thus impacting the point detected by the hardware [3]. Forlines et al. found that touching a target using a flat finger angle leads to larger offsets [12]. Wang and Ren found that finger posture and motion impact the size of the contact area [23]. Holz and Baudisch further showed that differences in finger roll resulted in offset [15].

2.2.2 Spread and Minimum Button Size. Vogel and Baudisch demonstrated that for rectangular shapes (using the centroid as a target) the minimum size of buttons was $10.5 \mathrm{~mm}$ [22]. In their experiment using cross targets within a visible circle, Wang and Ren [23] found that the diameter of circular shapes needs to be greater than $11.82 \mathrm{~mm}$ and square shapes need to be at least $11.52 \mathrm{~mm}$. Hall et al. [14] reported minimums button size of up 26mm for rectangular shapes. And Holz and Baudisch [15] reported a minimum of button size of $15 \mathrm{~mm}$ using cross shapes but that this value could be less if correcting for finger yaw pitch roll. These authors investigated the effect further [16] and observed that users touched a cross target by placing a fixed point located on top of their fingernails over the shapes. Users thus seem to use visual alignments. The same authors also note that the disagreement about minimum button sizes of these different studies cited above is possibly caused by differences in study conditions (e.g. finger orientation).

2.2.3 Effect of Shape Geometry. Roudaut et al. [19] investigated touch accuracy with different surface geometry. The authors looked at touch on curved surfaces. They also used cross targets, but the surface had different curvatures (concave to convex). They show that concave surfaces induced larger spread and larger offsets. Bacim et al. [1] looked at touch accuracy on deformable surfaces (also using cross shapes) and showed that the shape of the surface affects

\footnotetext{
${ }^{1}$ For clarity we will refer as target when it is only one point.
} 
touch selection accuracy for both touch position and deformation distance.

\subsection{Pointing vs. Touch Accuracy Studies}

Touch accuracy studies, our focus in this paper, are different from pointing studies. Pointing studies focus on the speed-accuracy tradeoff in pointing tasks while touch accuracy studies focus on where users aim at via a form of untimed docking task. The difference is that pointing studies are interested in the combination of movement and precision. Originating from Fitts' law, there is today a multitude of variations to encompass diverse interactive contexts [2, 17] There is particularly one from $\mathrm{Bi}$ et al. proposing an adapted model for pointing targets on a touchscreen [4].

Despite a large amount of pointing studies, few are looking at target geometries. Chen [6] changed the shape of keys in soft keyboard to increase typing speed. Grossman et al. [13] proposes a Fitts's Law model for 2D arbitrary shaped shapes acquired with a mouse input device. The model can compute the index of difficulty of any arbitrary 2D shape. The studies demonstrate the geometries of the shape impact pointing time. For example, for a shape looking like the "plus" sign, over $90 \%$ of selection points were within the horizontal region of the target, the other being in the vertical region. In a study performed with a pen as a pointing device, Cressman et al. [7] demonstrated that subliminal geometries influence pointing activity and suggested that users point differently depending on the shape geometry. These studies seem to highlight an effect of the geometry of the shapes, but they have only investigated it under the light of pointing studies. Additionally, none of them have looked at the particular case of touchscreen interaction.

\section{PRELIMINARY STUDY}

Our goal was to investigate how users think they touch 2D arbitrary shapes and identify touch strategies. We took inspiration from $\mathrm{Holz}$ and Baudisch's papers $[15,16]$, and used interviews to learn about users' mental models when touching non-rectangular shapes. This study focused on eliciting thoughts and qualitative data rather than devising quantitative metrics.

\subsection{Participants}

We recruited 12 participants ( 7 female, $M=25.82$ years, $S D=4.35$ ) from three different institutions ( 4 in UK, 4 in Singapore, 4 in France). They were all regular touchscreens users. The study lasted about 30 minutes per participant. No compensation was given.

\subsection{Task and Procedure}

Participants were provided with a printout of the shapes on a sheet of paper. They were instructed to touch the printout as if they were trying to acquire each shape as accurately as possible and as if they were on a touchscreen. We were very careful not to provide any hints about the way the shapes could be touched to avoid biases. We told them to act as if the shapes were on a touchscreen, but did not provide any other direction and did not answer any question that could influence their behavior. They were allowed to repeat the process as many times as they wanted before deciding on the final positioning of their fingertip on each shape. They were asked to think aloud during the process. Once they settled, participants drew

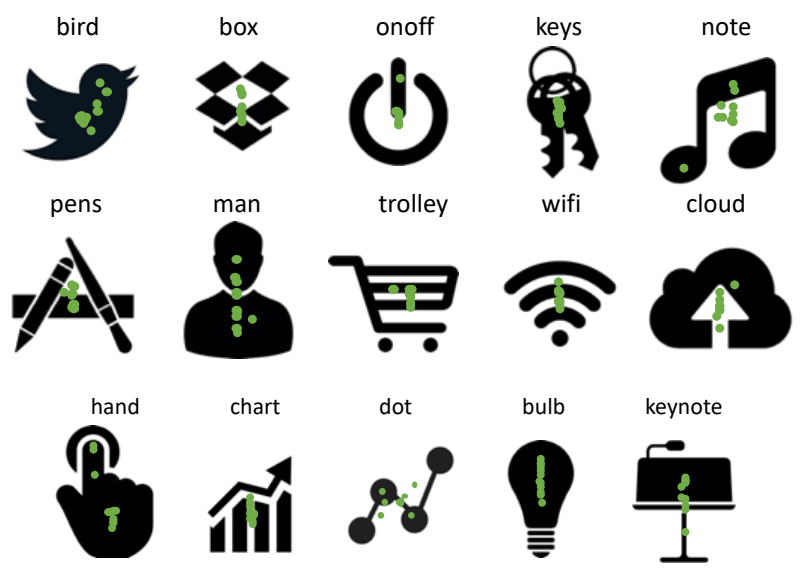

Figure 1: 15 shapes used in our qualitative study, green dots represent the position indicated by participants.

a dot on the paper to indicate the precise location they thought they would aim at when touching the shape. Then, we proceeded to a semi-structured interview during which the experimenter inquired about what part of the shapes they were trying to touch, why, and the strategy they used.

\subsection{Shapes}

We selected fifteen shapes (Figure 1). To do so, a large set of candidate shapes was created from a google search with the keyword "shapes set". Note that we could have also picked from a set of existing buttons but our contribution generalizes to any touchable elements so we used a rather general set through the google search. Each author individually reviewed the set and subjectively selected a subset of ten items. Then, all authors discussed and decided on a final selection of fifteen shapes. The selection criteria were to vary geometries, curvature, and weight distribution (e.g. holes, or asymmetries), to trigger different reactions, and discover various touch strategies. This first experiment being exploratory, selection criteria were broadly defined and left to interpretation. Generating shapes systematically would have had few benefits at this stage. Fifteen shapes were included as a compromise between the diversity of the shapes, and the duration of the experiment. We scaled them so that their bounding box was $18 \mathrm{~mm}$ wide, which is the average width of a human index fingertip [8].

\subsection{Design}

The experiment included fifteen shapes (Figure 1). The order of the shapes was randomized.

\subsection{Results}

We performed a thematic analysis [5] of the interviews. A first coder labeled the feedback with initial codes to characterize a method used for touching each shape. Then, with a second coder, they discussed and refined the codes, and identified the themes presented below.

Figure 1 shows the subjective touch location each participant settled for each shape. Some patterns are noticeable from this data, such as a tendency to ignore holes within shapes, or some tendency 


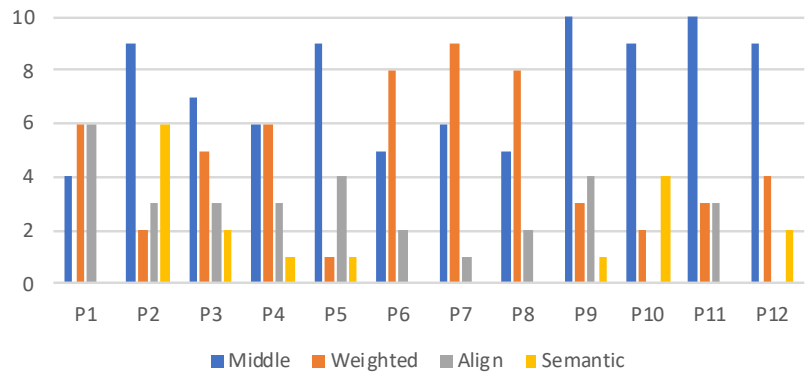

Figure 2: Number of times the different methods/themes were mentioned by our participants (P1-P12).

to aim where the shape is the densest, such as the top of the bulb. Participants feedback revealed four main strategies: "aiming at the middle" (45.9\% of the thematic analysis codes); "aiming where there is the most shape" (29.4\%), "aligning the fingertip with the shape features" (16\%) and "aiming at semantic features" (8.7\%). Figure 2 gives more details about the diversity of strategies reported by participants.

3.5.1 Aiming at the Middle (S1). The most used strategy was to find the middle of the shape and aim at it. But the term middle had several meanings for our participants as seen below with a selection of comments.

Centroid: "I use the centroid of the shape" (P11 cloud).

Middle: "kind of the center of the shape even if it is empty" (P1 trolley); "in the middle of the overall shape" (P2 chart); "the overall center (P3 chart)"; "middle of it as if it was one block without empty space" (P4 trolley); "right in the middle" (P6 note); "center of the whole shape" (P7 bulb).

Blob (circle/ellipse): "in the middle, like it is like a blob, even if there are empty parts (P2 box)"; "center of the blob, it does not matter if the head is small, I am mentally filling the empty space" (P5 man). "middle of the ellipse fitting (P9 dots)"; "center of circle fitting in the shape" (P12 note).

Bounding box: "center of the bounding box, in this case of a single note I would target either center or the circle on the note" (P7 note); "it needs to fit within the bounds of the shape" (P12 bird). In some cases (bounding box or ellipse fitting), we also observed that participants tilted their fingertip so that it matches the shape.

3.5.2 Aiming where the shape is the densest (S2). The second most mentioned strategy consists of locating the densest part of the shape and ignoring voids.

Densest part of the shape: this was described by a range of different words: "where the most of the volume is located (P1 hand)"; "the biggest part of the color" (P3 finger); "major area of filling" (P3 pens); "wilder part" (P4 hand); "bigger area of the bird, the more probably I will be in" (P4 bird); "the middle of the key is where the volume is" (P4 key); "I try to go for where there is more" ( $\mathrm{P} 5$ hand); "bottom is heavier" ( $\mathrm{P} 7 \mathrm{box}$ ); "better concentration of drawings" (P9 keynote); "center of gravity" (P9 hand); "more matter" (P10 note); "where the mass is the most" (P11 note); "in the chubby parts" (P12 bird); "round bits more filled" (P3 keys).

Ignore voids in the shape: participants chose to remove shapes details: "don't bother for the empty, I reach the top as there is more surface" (P5 wifi); "I removed the pointy parts" (P5 chart); "I ignore the top ring as it is small" (P6 keys); "I don't mind the trolley handle" (P6 trolley); "Ignore the little details" (P10 trolley); "the foot is not useful" (P10 keynote).

3.5.3 Aligning the Finger with the Shape Features (S3). The third type of strategy observed relates to the action of aligning the fingertip with a visible part of the shape. We observed different kinds of alignment strategies corresponding to the following codes:

Finding fingertip shapes: "it looks like my fingertip, so the top aligns with my nail" (P1 bulb); "I pressed where I was sure my fingertip fits entirely as I can see the shape outside" (P1 onoff); "in the middle it has a fingertip shape" (P2 onoff); "it fits my fingertip and align on the top" (P2 wifi); "put my fingertip in the middle of the circle" (P5 onoff); "I see the shapes as a big blob and I try to cover my finger with it" (P6 pens). "I tilt the finger and cover as much as possible with my finger" (P8 dots);

Finding axes and symmetry: "if I align the top of my fingertip on the top then I am sure the point will be inside" (P1 wifi); "if an axis is larger than my fingertip, I try to go at the center of it (and the other is not as important)" (P5 keynote); "Horizontally on the center, vertically in between the two part we can identify" (P7 chart). "The horizontal rectangle is more salient, and I can align" (P2 pens); "also align with the vertical stroke as to make sure that it is touched" (P7 onoff); "I use the center of the symmetry as guide" (P9 pens); "there is a triangle, I aimed the center of it and align my finger on it" (P4 pens). Looking at Figure 1, we see that participants mostly aimed at the center of the symmetry if there is one in the shape.

Not exceeding: participants also commented on using the trick of covering the shape entirely so nothing exceeds from under their fingertip: "I try to cover all the shape with my finger, so everything is under" (P11 dots); "my goal is to cover the whole shape with my finger" (P6 bird); "I can see the isolated point at the top when my fingertip covers the rest" (P10 dots).

3.5.4 Aiming at semantic features (S4). Our participants used semantic properties within the shape to decide where to point: "point around the heart of the man" (P2 man); "in the middle of the trolley as it is where you put stuff" (P2 trolley)"; "the arrow attracted me, so I put it at the top of the arrow" (P2 arrow). "The arrow attracted me" (P3 arrow); "in the top, because it is where the light comes from" (P2 bulb); "I feel bad to touch the head" (P3 man); "in the middle of the bird body but not the head" (P3 bird); "I aim at the head as it is more important" (P6 bird); "because I could not cover the whole shape, I aimed at the head because it is more important than the body" (P6 man); "Only the top because it seems more interactive" (P8 onoff). 


\subsection{Discussion}

The first insight from our study is that participants used different strategies to locate a point on the shape to aim at (the perceived input point). This point is often indicated as the center of a particular geometric element ( $75.3 \%$ of the time). We identified four different types of strategies: $\left(S_{1}\right)$ the participants searched for the center of the shape contour; $\left(S_{2}\right) 29.4 \%$ of participants focused on a particular part, with the "most content", and estimated its center. The two other main strategies consisted of mentally fitting a simple shape and assess its center, specifically $\left(S_{3}\right)$ a rectangle, or $\left(S_{4}\right)$ an ellipse.

We also found that participants used the visual features of the shape extending from under their fingertips to aim more accurately ( $16 \%$ of the time). Consequently, results differed whether the finger partially or completely occluded the shapes. For example participants with larger fingers, like P10 and P12, never mentioned alignment strategies. This led to interesting insights: when the shape was totally occluded participants aimed at what they perceived as the center of the shape. However, when they could see parts of the shape extending under their fingertip, participants also made use of visible cues to align their fingertip and achieve better precision.

Finally, we observed different behaviors when the shape had a semantic meaning ( $8.7 \%$ of the time). For example, some participants aimed at a meaningful location, such as the location of the heart or the head.

\subsection{Hypotheses and Research Questions}

In light of observations from our first study and clues from related work, we lay out the following research questions and hypotheses:

RQ: Among the four different strategies reported by our participants (S1-S4), which one is the most representative of touch accuracy on shapes with arbitrary geometries?

H1: The spread of input points is smaller when a shape is only partially occluded shapes during touch.

H2: Like in previous studies, a systematic offset should be observed. However, this offset does not vary with target size.

$\mathrm{H} 1$ comes from the findings that participants align their fingers with visible shape features extending from under their fingers as they come into contact. By aligning their finger with visible features, we hypothesize users can be more consistent. $\mathrm{H} 2$ originates from previous work on rectangular targets [3, 12, 15, 23], and our preliminary study.

\section{EXPERIMENTAL DESIGN PREPARATION}

Designing an experiment to test our hypotheses is not straightforward because: (1) we need to mathematically model the qualitative strategies extracted from our preliminary study; (2) we need to select a set of arbitrary shapes to study which maximize the distance between those mathematical models; (3) we needed to choose different size of arbitrary shapes to simulate the condition of "occluding completely the shape vs. not". We explain these three steps in details, i.e. how we generated nine mathematical models to reply to (1); how we used a computational approach to reply to (2) - this approach maximized distances between model predictions to generate 15 shapes (Fig. 4); how we used two different sizes of shape that are relative to the finger (ratio of the index fingertip 75\% and $125 \%$ ), which is atypical from conventional touch studies.

\subsection{Generating seven mathematical models from the qualitative touch strategies}

The strategies we identified in the first study are combinations of participants' descriptions of their mental processes. They are not algorithms with sequences of specific operations. Therefore, several interpretations lead to several distinct implementations (or models). We describe below our implementations, that we coded with the Python version of OpenCV 3.0 2 .

4.1.1 Contour Centroid. With Contour Centroid strategy, participants took into account the boundaries shape. The variations lie in the way they considered the space around the shape. Our two implementations of this strategy compute the centroid of a contour as opposed to the whole area of the shape. The two variations consist in considering $\left(S_{1 a}\right)$ the contour of the shape itself (findContours, [21]), or $\left(S_{1 b}\right)$ the convex hull of the shape (convexHull, [20]).

4.1.2 Large Cluster Centroid. Participants who followed this strategy focused on parts of the shape with more pixels. In other terms, they aimed at the biggest clusters of pixels. $\left(S_{2}\right)$ we implemented this strategy by computing the centroid of dark pixels, that is to say, the average coordinates of these pixels. Dense clusters of pixels pull the centroid in their direction.

4.1.3 Fitting Rectangle. Some participants attempted to align body parts, a finger or a nail, with the shape. The strategy we associated consists in fitting a rectangle to the shape and we propose two implementations. $\left(S_{3 a}\right)$ computes the center of the bounding box of the shape (boundingRect). The rationale for this is that it matches the way shape pointing is typically coded. $\left(S_{3 b}\right)$ matches the minimum area rectangle and allows for rotations (minAreaRect). Note that the boundingRect $\mathrm{s}$ aligned with the $\mathrm{x}$ and $\mathrm{y}$ axes. The minAreaRect $\mathrm{s}$ smaller because it allows rotations.

4.1.4 Fitting Ellipse. This is a variation of the previous one. $\left(S_{4 a}\right)$ consists of searching the minimum enclosing circle of the shape (minEnclosingCircle). It refers to a simplified view of the fingertip. $\left(S_{4 b}\right)$ is a variation of $\left(S_{3 b}\right)$ that fits the shape with an ellipse. The implementation uses the fitEllipse function [11]. This algorithm requires at least five points, therefore this strategy cannot be computed with the simplest shapes.

\subsection{Generating 15 shapes maximizing distance between mathematical models}

To select shapes to study, we choose to create a subset of shapes maximizing the distance between our models, which in turn increases the chance to find the signal within the noise. We were indeed constrained by the intrinsic limitations of experimental design and statistical analysis, and only include a fixed number of shapes while also maintaining enough repetitions and factoring in 2 sizes. So to test our hypotheses we chose a systematic shape generation to ensure the difference between the seven models would be maximized.

\footnotetext{
${ }^{2}$ The source code will be made available when this work will be published
} 
Note that another solution is to use shapes used in former work such as Grossman et al. [13], investigating mouse pointing for nonrectangular shapes. However, almost all of these shapes have one or more symmetries (Figure 3). Therefore they are special cases, and are not suitable enough to distinguish between the models: the perceived center for the strategies we identified are close from another. This set is thus not ideal to investigate our touch strategies. Besides, our study focuses on touch, which involves a larger error spread than with the mouse, thus increasing the necessity to have shapes with different input points for each model.

We selected 15 shapes (Figure 4) whose geometries were different enough so that the input points for each of our touch metrics would be further apart from each other. The reason behind this choice was to increase the chances to validate or invalidate the touch metric as the finger touch accuracy is known to be low [15]. We implemented a script that computes the distance between the output of our models. Our script works in three steps. First, it normalizes each shape to a standard size $([0-1])$. Second, it computes the output of each of our models for each shape. Third, it extracts the shapes that maximize the Euclidean distance between those input points. To do so, the script computes the average of the Euclidean distance between the outputs of each pair of strategies for each shape. More specifically, given $c_{1}, \ldots, c_{7}$ the centers predicted by our seven strategy implementations for a specific shape, and $C(7,2)=21$ the number of possible pairs of models, the metric is:

$$
m=\frac{\sum_{1 \leq i<j \leq 7} \sqrt{\left(c_{i} \cdot x-c_{j} \cdot x\right)^{2}+\left(c_{i} \cdot y-c_{j} \cdot y\right)^{2}}}{C(7,2)}
$$

The average of the fifteen shapes selected by this process is $9.02 \%$ $(\min =0.69 \%, \max =17.6 \%)$.

The first part of our script takes multiple square shapes as an input, and thresholds the pixels so that they are either considered as shape or background. Then it computes the $\mathrm{x}-\mathrm{y}$ coordinates of the input point for each models. We ran this script on a set of 374 shapes found on traditional user interfaces (a random selection from google image using "shapes set" as the keyword). The second part of our script loads the CSV file generated and computes the average distance between each model by running pairwise comparisons. This script allows us to rank our shapes according to the differences between each model. From here, we extracted the fifteen shapes (Figure 4), which maximize the average distance between model outputs. Figure 5 shows the mean distance between the strategies for all the shapes we investigated. For many, the output of our models tended to overlapped. We picked the 15 shapes with the least overlap, in the far right tail of this distribution.

\subsection{Choosing two relative size of shape instead of absolute ones}

Most studies looking at targeting, pointing, or touch make use of different shape sizes. These sizes are normally absolute, i.e. they can be given as a measure in millimeters, which does not vary between participants. However, in the case of our study, we are highly interested in comparing phenomenon where the finger occludes totally or only partially a shape. In such a case, the size of the participant's fingertip becomes a confounding factor that must be treated separately. This is the reason why we chose relative shape sizes that were $75 \%$ and $125 \%$ of the distal phalanx width of the index fingertip of each participant. It thus means that we measured the participants' fingertips before starting the experiment. This measure was used to compute the two sizes of shape that were thus different for each participant. What was constant among participants was the two-scale factors. We thus report the size of finger size later in the experiment (Figure 6).

Note that using relative size shapes is not common but was needed to test our hypotheses that users have different strategies depending on whether or not the features would be visible from under the finger. Using a fixed size like in traditional studies would introduce a confounding factor (e.g. an $8 \mathrm{~mm}$ shape be invisible under a large finger but not under a small one). This also explains why we then normalized our data (further explained in 5.6), i.e we scaled the participants' fingers and input to the same standard size of a finger to compare the results.

\section{CONTROLLED EXPERIMENT}

We gathered touch data on 15 arbitrary shapes and two relative sizes. We then compare this data with our models' predictions to identify the one best able to predict pointing behavior. Note that this experiment may misleadingly seem related to well-known speed-accuracy results and Fitts' law $[10,17]$ that show users point faster on larger targets as less accuracy is required. Despite similarities, we did not implement a Fitts task. We did not investigated motor control, but the mental model and user strategies. We measure spread that may result from different strategies or inconsistent interaction, not accuracy as a function of movement speed. Participants were explicitly told to use all the time they needed.

\subsection{Apparatus}

Like in previous work [15] we used a capacitive sensing technology to detect touches. We used a Samsung Galaxy S3 Tab, with a 9.7" capacitive touchscreen to display the shapes and record the user input. The tablet has a resolution of $2048 \times 1536 \mathrm{px}$, thus a pixel width is $0.096 \mathrm{~mm}$. We chose this tablet because unlike Apple devices, there is no automatic correction of the touch input, i.e. it is computed as the centroid of the contact area. We could not find information about how the capacitive grid technology underlying the hardware we used treated the data. Therefore, to ensure the data was consistent and to remove potential noise from the hardware, we placed the shape always in the same position on the touchscreen. Note that we choose not to use a pen as Holz and Baudisch [16] suggest users use features of their finger when touching precisely a target.

\subsection{Task}

During each trial, participants used their dominant hand to press a start button at the bottom of the screen before touching a shape. Following previous approaches $[9,12]$ participants had to validate the touch input acquisition using an external action: tapping one of the two buttons displayed the right or left side of the interface with their non-dominant hand (Figure 6). To prevent repeated mechanical movements, the distance between the start button and the shapes was randomized. The location of the shape on the screen 


\section{$\Delta \circlearrowleft \models \omega \square \square \omega \backsim \sigma$ \\ Figure 3: Shapes used in Grossman et al.'s pointing with a mouse study [13].

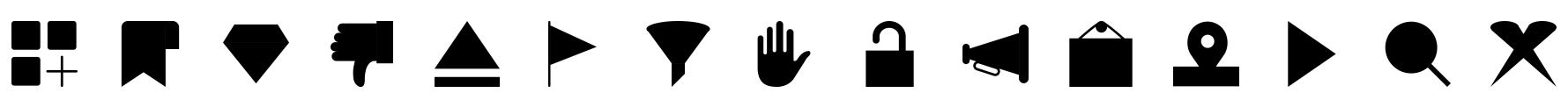

Figure 4: Shapes systematically selected by our script maximizing distances between model predictions.

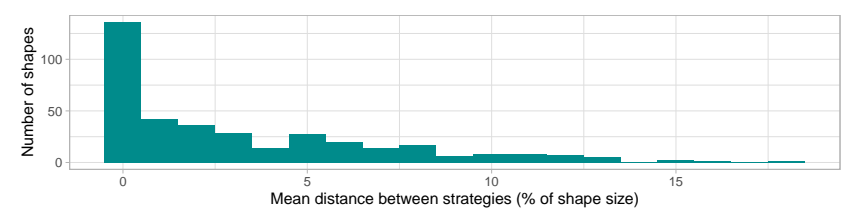

Figure 5: Distribution of the mean distance between strategies for all the shapes used in our extraction process. We used shapes at the far right to maximise the distance between strategies.

was randomized with the start button position chosen in a $50 \times 50$ px around the center of the screen.

\subsection{Procedure}

With the help of the experimenter, participants measured their index fingertip by positioning it on a user interface displayed on the tablet (Figure 6 a). The fingertip was measured from the first joint fold to the top, and from left to right. Then, participants opened the trial interface (Figure 6). They were allowed to take breaks between trials and were instructed to be as accurate as possible and to take all the time they needed to decide on the most relevant touch input point. To avoid any bias, we provided no direction on how to touch the shapes, for example how to deal with geometric specificities like holes. Due to the simplicity of the task and the speed requirements, no practice trials were required. The experiment lasted approximately 30 minutes per participant. Note that we provided instructions to ensure that the task in this experiment was understood the same way as in our initial experiment. In both studies, participants were told to take as long as they needed.

\subsection{Participants}

A total of 12 right-handed volunteers participated in the experiment ( 5 females, 18 -33 years-old, $M=20.6, \mathrm{SD}=4.1$ ). The finger sizes of our participants are shown in Figure 1. The mean of the fingertip height was $9.4 \mathrm{~mm}[\min 8.4 \mathrm{px} \max 11.1 \mathrm{px}](\mathrm{SD}=1.0 \mathrm{~mm})$ and the mean of the fingertip width was $6.1 \mathrm{~mm}[\min 5.1 \mathrm{~mm}, \max 7.0 \mathrm{~mm}$ ] $(\mathrm{SD}=0.6 \mathrm{~mm})$.

\subsection{Experimental Design}

We used a repeated-measures within-participant design with two independent variables: Shape (15 values, see Figure 4) and Size (ratio of the index fingertip at $75 \%$ or $125 \%$ ). The size was included to control and examine the effect of shape occlusion during touch.
As a result, it is defined as a factor of the participant's fingertip instead of absolute values. Shape and size orders were randomized. We recorded 15 shapes $\times 2$ sizes $\times 18$ repetitions $=540$ trials per participant.

\subsection{Results}

We removed outliers more than three standard deviations away from the mean ( $0.34 \%$ of the data). Aggregated data points are shown in Figure 7. This figure helps to see the trends. We first look at how consistent participants were by examining the spread of the touch location. Then, we investigate our models, and how efficient they are at predicting our participants' behavior. Because the effective sizes of shapes are proportional to the size of the participant's fingertips, we measured a normalized input point between 0 and 1 rather than pixels or millimeters. For example, a participant input located at $(0.5,0.5)$ would be in the middle of the shape's bounding square, and negative values or values larger than 1 indicate location outside this square.

5.6.1 Spread. Spread measures how far apart the points are. We calculated it as the average normalized distance of each input point to the mean touch location. We applied a cube root transform to normalize its distribution (confirmed by Shapiro-Wilk test). We applied Greenhouse-Geisser sphericity correction when needed, correcting both $\mathrm{p}$-values and degrees of freedom.

We found a main effect of Size on spread $\left(F_{1,11}=74.8, p<0.05\right)$ but none for Shape nor interaction Shape $\times$ Size. $75 \%$ sized shapes $(\mathrm{M}=0.1686,95 \% \mathrm{CI}[0.1646,0.1726])$ had significantly less spread than the $125 \%$ ones $(M=0.1951,95 \%$ CI $[0.1905,0.1998])$. If we take that the human size averages between $16-20 \mathrm{~mm}$ [8] it means that the spread with $75 \%$ sized shapes is around $2.7 \mathrm{~mm}$ and $3.4 \mathrm{~mm}$, while it is between $3.12 \mathrm{~mm}$ and $3.9 \mathrm{~mm}$ for $125 \%$ sized shapes. This goes against our assumption that shapes extending from under the fingertip would help the participants to be more consistent in their touch strategy (by letting them align their fingers with the visual clues extending from under their fingertip). On the contrary, the results show that large shapes had larger touch variation, most likely because the larger size required less precision.

5.6.2 Model Precision. Model Precision measures how efficient a model is at predicting users' behavior. It is calculated for each of our seven models as the average normalized distance from input point to model prediction. A non-parametric Friedman test was used to compare the precision of the models. Fig. 8 shows the distance-toinput of each model's prediction. Overall, the bounding box model best-modeled participants' input both for $75 \%$ and $125 \%$ shapes. 
a)

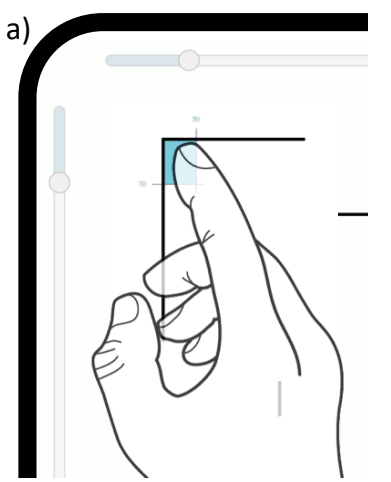

b)

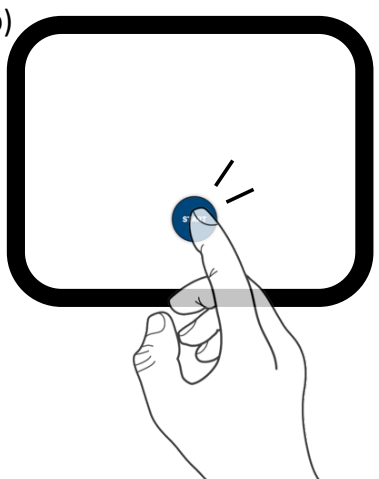

c)

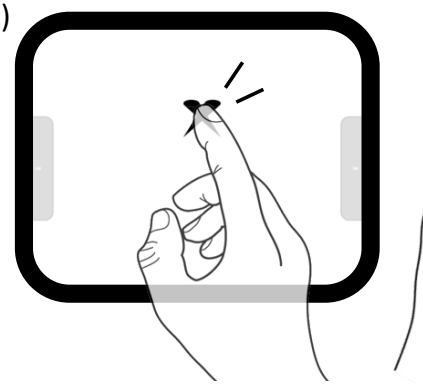

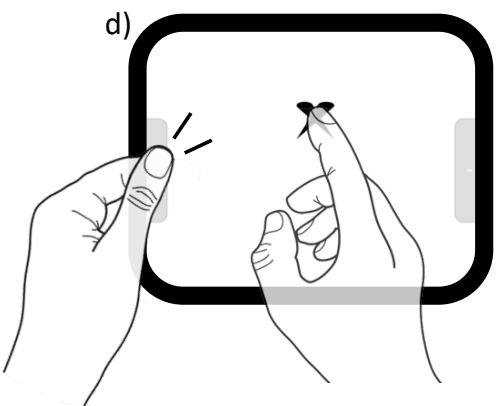

Figure 6: (a) at the beginning of the study, the participants' fingertips were measured on the tablet; a trial started by pressing (b) a start button before pressing (c) the shape; the participants confirmed the location on the shape by pressing the side buttons with their non-dominant hand.

Table 1: Participants fingertip sizes as measured at the beginning of the experiment.

\begin{tabular}{r|cccccccccccc} 
Participant ID & P1 & P2 & P3 & P4 & P5 & P6 & P7 & P8 & P9 & P10 & P11 & P12 \\
Fingertip Height $(\mathrm{mm})$ & 21 & 17 & 17 & 17 & 18 & 20 & 22 & 18 & 17 & 18 & 21 & 18 \\
Fingertip Width $(\mathrm{mm})$ & 13 & 12 & 11 & 10 & 11 & 11 & 12 & 11 & 13 & 12 & 14 & 13
\end{tabular}
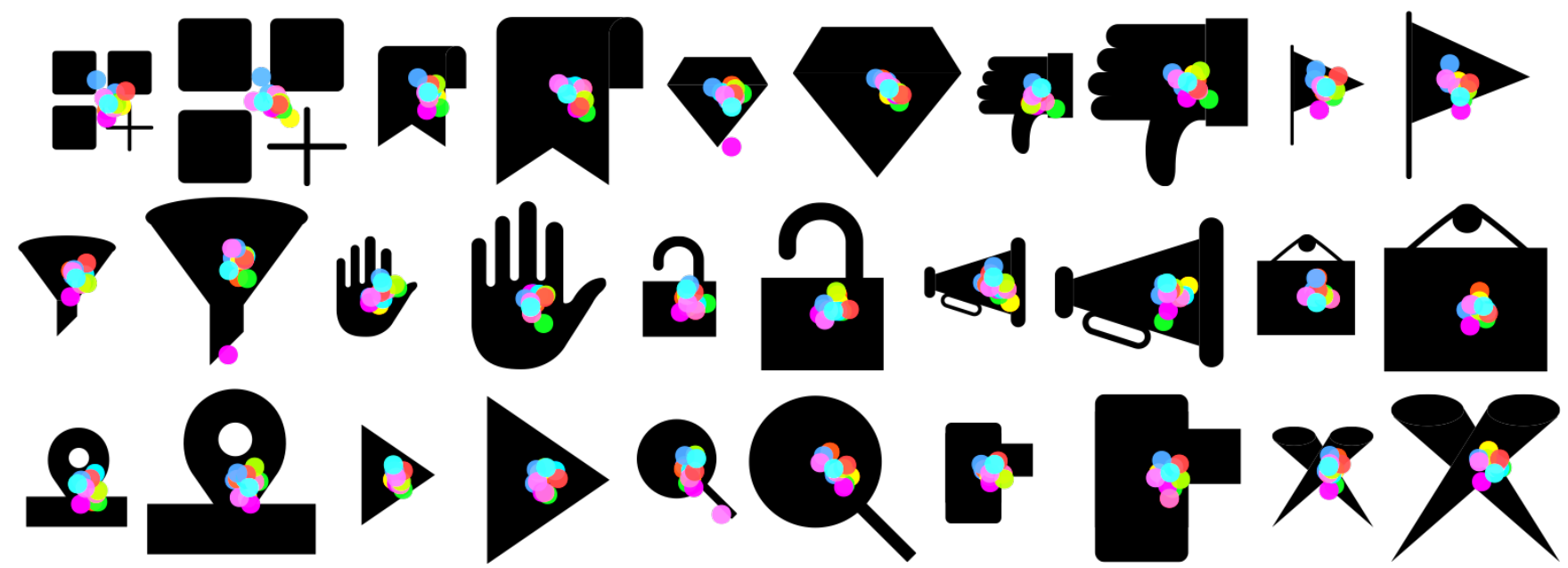

Figure 7: Aggregated and normalized results showing where participants aimed at the different shapes depending on their sizes. Each point represents a participant.

However, no prediction was perfect, and we observed a systematic bottom-directed deviation from the input occurring for each model. A similar systematic offset was already observed in previous work and corresponds to the direction of the fingertip spreading out onto the surface.

We used a Wilcoxon sign rank test using Bonferroni correction to compare model predictions. There was a statistically significant difference in precision depending on size $\chi^{2}(6)=2816.52, p<0.05$ : $75 \%$ shapes had a larger offset than $125 \%$ shapes, most likely due to greater occlusion. We found that all were significantly different except the $\left(S_{4 b}\right)$ ellipse vs. the $\left(S_{1 a}\right)$ polygon center; the $\left(S_{1 b}\right)$ convex hull vs. the $\left(S_{3 b}\right)$ oriented box; the $\left(S_{4 a}\right)$ circle vs. the $\left(S_{3 b}\right)$ oriented box. We also checked that the same effects occurred for each shape size condition independently. Our results thus demonstrate that the bounding box model is the most representative of the users' behavior across the two sizes of shapes used in the study, and across all shape geometries.

5.6.3 Summary. We found an answer to $\mathbf{R Q}$ in that the bounding box model is the most representative of touch on arbitrarily shaped shapes. We did not find any significant differences for the types of model used depending on the size of the target. We invalidated $\mathbf{H 1}$ in that the spread is larger with large shapes contrary to what 


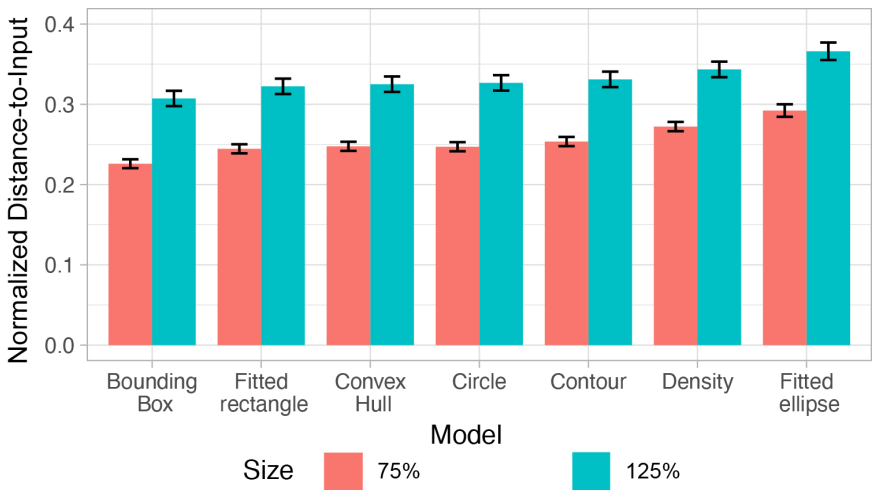

Figure 8: Distance from each model prediction to the input points with the two different shape sizes. To have the real size multiply the unit by the fingertip size $(\mathrm{e} . \mathrm{g} .16 \mathrm{~mm}$ to $20 \mathrm{~mm}$ ).

we assumed, suggesting users may not align their finger with the shape to make their repetitive hits more accurate. Like previous work, we observed a systematic offset (H2) but found this offset was further away from the bounding box center when the shapes were totally occluded compare to partially occluded.

\section{DISCUSSION}

We discuss here our findings as well as our limitations and future works.

\subsection{Bounding box model is the most representative}

Our results identify the bounding box model as the most representative. This suggests counter intuitively that the geometries of shapes can be ignored, which includes complex shapes like the one often found in games or drawing applications. In the context of icons or buttons, this result means that designers that are not secluded to rectangular shapes and instead can make use of many different geometries. Visual feedback indicating the touchable area does not even seem necessary as users are particularly efficient at mentally determining the center of the bounding box.

However, contrarily to pointing on shapes with a mouse and a cursor, we observed users tend to ignore holes or voids within shapes when touching shapes. Grossman et al. [13] showed that users tend to avoid holes when pointing on a shape with a mouse. Our experiment suggests that, with touchscreen, users tend to see a shape as a whole and to perceive it contained within a box. Whether this is an intuitive or learned behavior remains for future work. These results have particular consequences for user interfaces that would expect users to select a shape through another one, as is sometimes encountered in slide builders, or drawing applications.

\subsection{Spread is larger but offset is smaller on partially occluded shapes}

Participants appeared to be significantly more precise when touching shapes completely occluded by their fingers. This larger spread on larger shapes suggests that users do not make use of the visible features of a shape when touching it. This explicitly goes against the perception of our participants as reported in our first study. We found a correlation between the size of the shape and spread: our results show a confidence interval of [12.8-16 mm] for input spread on $75 \%$ sized shape, and [13.5-16.8 $\mathrm{mm}$ ] for $125 \%$ sized shape. This is arguably similar to the range already observed in the literature with rectangular shapes, but higher than with the results reported when tapping on crosses $(15 \mathrm{~mm})$ [16]. One explanation could be that as participants have more room to place their fingers on larger shapes, they struggle to identify one particular target. This tends to indicate that the larger a shape is, the more difficult it is to maintain consistency when touching it. On the contrary, crosses as used in previous studies exhibit a precise target and leaves no room for interpretation. One other reason could simply be that participants have less chance to miss the shape, and as a result, make less effort and demonstrate less precision when selecting a target.

On the other hand, we found the offset was larger when the shape was completely occluded by the participant's finger. In other terms, the distance from the finger to the center of the bounding box was smaller on shapes that were only partially occluded. This result gives designers more precision when attempting to predict how users will touch shapes depending on the size of the shape, and in particular how occluded it will be.

\subsection{Preliminary Study Results versus Controlled Study Results}

The verbalization of participants revealed different strategies are used to choose how to touch shapes of different geometries and sizes. Both our preliminary study and controlled study show that participants tend to aim at the center of a shape. However, our controlled study did not confirm that the weighted centroid (accounting for $29.4 \%$ of the comments in our preliminary study) was a prominent strategy with a touchscreen. On the other hand, participants of our preliminary study indicated they attempted to align their fingers with non-occluded features of the shape. However, our controlled study does not confirm this behavior, as shown in our input spread analysis and it is unclear why we observed this disparity. Consequently, it appears users are often mistaken in the strategy they think they use to interact with touch surfaces: while users tend to believe they make use of advanced touch strategies, they do tend to simply aim at the center of their target.

Another explanation could be that despite our optimization algorithm, the set of shapes we used did not provide enough variations. It is also possible that users do rely on specific strategies, but show little consensus between each other. As a result, the center emerges as a reliable one-fits-all model, but it may be possible to develop more precise per-user models. Finally, it is also possible that when using a touchscreen, our participants reverted to behavior learned from their past experiences. Further studies are needed to investigate these hypotheses.

\subsection{Limitations and Future Work}

Our study only focuses on two relative shape sizes. These sizes were chosen to investigate the effect of the shape's occlusion by the finger: $25 \%$ smaller and 25\% larger than the participant's index finger. However, the effect of larger or even smaller shape sizes remains 
unclear. For example, how do people aim at sizes up to four times the size of their finger? Do they make use of any particular strategy, still aim at the middle, or go for the closest point regardless of its location within the shape? Do they still ignore holes? With large shapes, it is also possible that certain zones become more salient than others, leading users to a different strategy that they would have used with a smaller shape. This paper opens opportunities to further explore these research questions.

One possible limitation of our studies is that in study 1 we used paper while in study 2 a touchscreen. This is coherent with standard research methodologies using mock-ups for initial observations and our second experiment builds on and validates our preliminary findings. But, like in other research, there are certain limitations of using paper mockups which are low-fidelity systems, and further investigations could be needed to check whether the support used prime participants into a particular action such as pointing explicitly within the bounding box. One extension of this work could be to compare two sizes of shapes with these two different apparatus to check if there is a difference in behavior.

The choice of shape we used in our studies is also critical. Our goal was to pick shapes that were ecologically valid, i.e. that we can find on various interfaces. But we also needed to find shapes different enough. These double constraints led us to pick from a shape set found online combined with our automatic extractor, which we believe is a good trade off. However, it is possible that some unusual types of shapes, not considered in the present work, lead to different results. Additionally, the metric we used to select our shapes in study 2 was sufficient to spread centers predicted by the models we implemented sufficiently far apart for some shapes. Other metrics may lead to other results, suggesting other shapes that favor another strategy. This research topic is fundamentally incremental, and we hope it will stimulate future studies.

In addition to the points above, our future works also include other directions. First, our results exclude the case of shape with semantics which we think would be relevant to study. We also would like to study shapes with more extreme geometries (e.g. with one axis disproportionate) to see if this had any effect on touch accuracy. Our work also only focuses on black on white shapes and adding colors, patterns and effects is a direction we are keen to explore. Finally, other factors would have also been interested to study, e.g. the physicality of targets.

Finally we would also like to note that we used the term "arbitrary shapes" in this paper and during the studies rather than "icons of arbitrary shapes". We did this to avoid priming the participants into understanding those shapes as widgets with a bounding box. In simple words our work makes a valuable contribution in confirming that users perceive icons as a box and designers do not need to worry about the shapes, but of course further studies need to be done to understand if our results apply to any arbitrary shaped interactive elements.

\section{CONCLUSION}

This work contributes to understanding touch on interactive surfaces. We investigated how the geometry of shapes impacts touch input. From the initial feedback of participants, we emitted hypotheses, extracted potential touch strategies, and built corresponding mathematical models. During our preliminary study, participants reported leveraging visible shape geometries to align their fingers, but our controlled study instead showed they tended to ignore shape geometries, and simply aim for the center. Indeed, a bounding box model best fitted their behavior. These studies, expose relevant insights that expand our understanding of human touch behavior and can help designing touchscreen interfaces.

Rectangular targets on rectangular displays have been the gold standard since the $80 \mathrm{~s}$, but this trend is increasingly challenged as designers explore novel platforms and new ways to interact with our information systems. User interfaces are not restricted to flat and rigid surfaces anymore. Wearables, virtual reality, or even organic user interfaces are all examples of such systems. Understanding how fundamental interactions, like touch, operate in these new interactive spaces is essential for the success of these platforms. By doing so we can gather empirical evidence, and create human models that can guide designers when creating new interfaces. This is ambitious; the space is large, and characterizing touch on different topologies is complex. This research is the first step in this direction (flat surface, geometrical shapes), and we believe it can inspire other researchers to expand our knowledge with more diverse topologies.

\section{ACKNOWLEDGMENTS}

This work was made possible by Daniel Vogel, NSERC Discovery Grant 2018- 05187, the Canada Foundation for Innovation Infrastructure Fund 33151 "Facility for Fully Interactive Physio-digital Spaces," Ontario Early Researcher Award ER16-12-184. It work was also supported by the Engineering and Physical Sciences Research Council (EP/P004342/1) and the Faculty of Engineering of the University of Bristol. We also thank Sylvain Malacria for his insights and participation to some of our discussions.

\section{REFERENCES}

[1] Felipe Bacim, Mike Sinclair, and Hrvoje Benko. 2013. Understanding touch selection accuracy on flat and hemispherical deformable surfaces. In Proceedings of Graphics Interface 2013. 197-204.

[2] Nikola Banovic, Tovi Grossman, and George Fitzmaurice. 2013. The effect of time-based cost of error in target-directed pointing tasks. In Proceedings of the SIGCHI Conference on Human Factors in Computing Systems. 1373-1382.

[3] Hrvoje Benko, Andrew D Wilson, and Patrick Baudisch. 2006. Precise selection techniques for multi-touch screens. In Proceedings of the SIGCHI conference on Human Factors in computing systems. 1263-1272.

[4] Xiaojun Bi, Yang Li, and Shumin Zhai. 2013. FFitts law: modeling finger touch with fitts' law. In Proceedings of the SIGCHI Conference on Human Factors in Computing Systems. 1363-1372.

[5] Virginia Braun and Victoria Clarke. 2006. Using thematic analysis in psychology. Qualitative research in psychology 3, 2 (2006), 77-101.

[6] Hsi-Jen Chen. 2014. Changed shape of key: an approach to enhance the performance of the soft keyboard. In Proceedings of the 16th international conference on Human-computer interaction with mobile devices \& services. 447-452.

[7] Erin K Cressman, Ian M Franks, James T Enns, and Romeo Chua. 2007. Online control of pointing is modified by unseen visual shapes. Consciousness and Cognition 16, 2 (2007), 265-275.

[8] Kiran Dandekar, Balasundar I Raju, and Mandayam A Srinivasan. 2003. 3-D finiteelement models of human and monkey fingertips to investigate the mechanics of tactile sense. F. Biomech. Eng. 125, 5 (2003), 682-691.

[9] Sacha N Duff, Curt B Irwin, Jennifer L Skye, Mary E Sesto, and Douglas A Wiegmann. 2010. The effect of disability and approach on touch screen performance during a number entry task. In Proceedings of the Human Factors and Ergonomics Society Annual Meeting, Vol. 54. SAGE Publications Sage CA: Los Angeles, CA, 566-570.

[10] Paul M Fitts. 1954. The information capacity of the human motor system in controlling the amplitude of movement. Fournal of Experimental PSychology 74 (1954), 381-391. https://doi.org/10.1037/h0055392 
[11] Andrew W Fitzgibbon and Robert B Fisher. 1995. A buyer's guide to conic fitting. In Proceedings of the 6th British conference on Machine vision (Vol. 2). BMVA Press, 513-522.

[12] Clifton Forlines, Daniel Wigdor, Chia Shen, and Ravin Balakrishnan. 2007. Directtouch vs. mouse input for tabletop displays. In Proceedings of the SIGCHI conference on Human factors in computing systems. 647-656.

[13] Tovi Grossman, Nicholas Kong, and Ravin Balakrishnan. 2007. Modeling pointing at targets of arbitrary shapes. In Proceedings of the SIGCHI conference on Human factors in computing systems. 463-472.

[14] Anthony D Hall, James B Cunningham, Richard P Roache, and Julie W Cox. 1988 Factors affecting performance using touch-entry systems: Tactual recognition fields and system accuracy. Fournal of applied psychology 73, 4 (1988), 711.

[15] Christian Holz and Patrick Baudisch. 2010. The generalized perceived input point model and how to double touch accuracy by extracting fingerprints. In Proceedings of the SIGCHI Conference on Human Factors in Computing Systems. 581-590.

[16] Christian Holz and Patrick Baudisch. 2011. Understanding touch. In Proceedings of the SIGCHI Conference on Human Factors in Computing Systems. 2501-2510.
[17] I Scott MacKenzie. 1992. Fitts' law as a research and design tool in humancomputer interaction. Human-computer interaction 7, 1 (1992), 91-139.

[18] Anne Roudaut, Stéphane Huot, and Eric Lecolinet. 2008. TapTap and MagStick: improving one-handed target acquisition on small touch-screens. In Proceedings of the working conference on Advanced visual interfaces. 146-153.

[19] Anne Roudaut, Henning Pohl, and Patrick Baudisch. 2011. Touch input on curved surfaces. In Proceedings of the SIGCHI Conference on Human Factors in Computing Systems. 1011-1020.

[20] Jack Sklansky. 1982. Finding the convex hull of a simple polygon. Pattern Recognition Letters 1, 2 (1982), 79-83.

[21] Satoshi Suzuki and Keiichi Abe. 1985. Topological structural analysis of digitized binary images by border following. Computer Vision, Graphics, and Image Processing 30, 1 (1985), 32-46.

[22] Daniel Vogel and Patrick Baudisch. 2007. Shift: a technique for operating penbased interfaces using touch. In Proceedings of the SIGCHI conference on Human factors in computing systems. 657-666.

[23] Feng Wang and Xiangshi Ren. 09. Empirical evaluation for finger input properties in multi-touch interaction. In Proceedings of the SIGCHI Conference on Human Factors in Computing Systems. 1063-1072. 